\title{
DEVELOPMENT OF COOPERATIVE AGRO-CREDIT SYSTEM IN GEORGIA
}

\author{
PAATA KOGUASHVILI \\ Doctor of Economic Sciences, Professor \\ Georgian Technical University, Academician of the Georgian Academy of Agricultural Sciences, Georgia \\ paata_koguashvili@hotmail.com
}

\section{DAVID MAMUKELASHVILI}

Doctor of Economics, Georgia

dmamukelashvili@gmail.com

Abstract. The work deals with the progressive system social and economic changes to be implemented in the given historical period in Georgia, which are based on the fundamentally different approaches to the economic policy. This is to lead an intensive rise in the population's income and in the national production output, mass employment and will ensure the formation of an environment targeted to a long-term development of entrepreneurship.

A special role for the social-economic development of a society is ascribed to the use of inclusive forms of entrepreneurship, which conditions, in turn, the necessity of the cooperation sector development - an important component of the modern economy. The cooperation projects proper have the viability and sustainability basis, which, together with economic selfgovernment, is conditioned by the high level of production concentration. Only by mass production (through reduction of prime cost, generation of added value in the uniform production cycle, constant, effective rather than impulsive operation). It would be possible to ensure competitive production and accumulation of a definite part of revenues from its sales for reproduction, without which the economic system development and the spill-over effects could not be achieved. Therefore, cooperation projects have objective bases of real viability and sustainability. The advantage of the given model consists in a complex nature of the main directions, ensuring thus the best conditions for approaching the optimum functioning of enterprises.

The future of the Georgian village depends on the correct analysis and assessment of the key problems, without which the establishment of progressive views concerning its development and the working out of relevant projects would be impossible. The finding out of an organizational and legal form, most complying with the social and economic mission of the State in the village most prioritized in terms of the governmental support is of vital importance in the proper policy establishment.

The work deals with the impact of agricultural cooperation and cooperative agro credit system on the entrepreneurial development of the population directly engaged in the agricultural sector and agricultural production. It also considers the major grounds for the development of agricultural cooperation in Georgia, describes the current economic state of the local agricultural production and rural population and makes an analysis of the increased migration causes and the organizational setup of agricultural activity. Thanks to analysis of the current social-economic state in Georgia and the experience of the successful countries, it is proved that the entrepreneurial activation of the population is best achievable by an active support of the cooperative sector, which will be directly reflected in the reforms to be implemented in the country regions and by using diverse forms of cooperation will enable mass employment of the population and intensive raising of national production output. A special and irreplaceable role of agricultural cooperation in overcoming rural poverty, in the stepping up of the entrepreneurial activity of the population and in implementing the complex process of forming a healthy middle class has been justified.

The legal regulation of the agricultural cooperation process and the industrial regulations represent the main component identifying the organizational form of an agricultural cooperative, because the introduction of these rules proper creates an economic environment, in which a high degree of return of the labour resources of the population directly engaged in the agricultural production rather than the prospects of gaining dividends from the material resources contributed to the share fund represents the factor determining the rural entrepreneurial activity. The industrial regulations of the cooperative system represent the basis for minimizing the production risks, rising economic efficiency, stability and viability.

The Georgian village owing to disregard and improper social-economic policy carried out for years has found itself in a very humble state. Under such conditions, the recovery by the complex and dissimilar system without the respective protective mechanisms and support is impracticable. Therefore, the establishment of a qualitatively new policy of inclusive socialeconomic development in respect to existing circumstances is a must, for which the inside and outside poverty-determining factors should be identified and the mechanisms of overcoming them be worked out. For conducting a viable national policy 
should be categorically defined that the hard economic standing of the rural population at the current stage is a problem of special urgency and the national policy should be mostly based on the given reality. For this, the special impact of agricultural cooperation and of cooperative agro credit system on the country's development should be adequately thought out, and the State should, like many developed countries, undertake and fulfil the coordinating, organizing and supporting function of the sustainable and forced development of this system.

KEYWORDS: SMALL POPULATION, ENTREPRENEURIAL ACTIVATION, ECONOMIC CHANGES, PROCESS COORDINATOR, AGRICULTURAL COOPERATION, AGRO-CREDIT SYSTEM.

For citation: Koguashvili, P., Mamukelashvili, D. (2020). Development of Agro-credit System in Georgia. Globalization and Business, 10. 180-184. (In Georgian) https://doi.org/10.35945/gb.2020.10.022

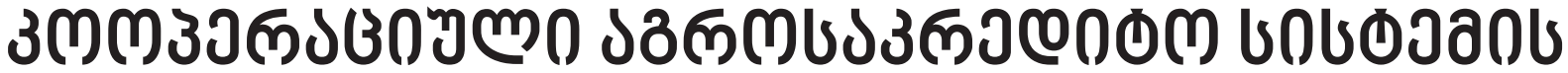

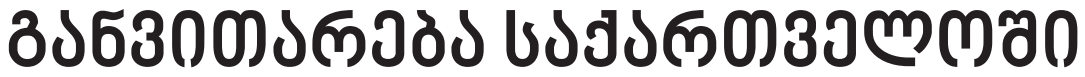

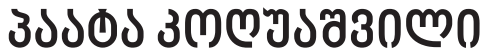

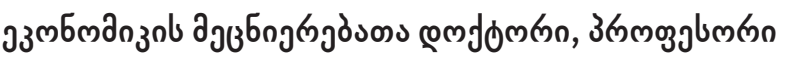

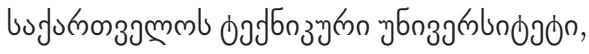

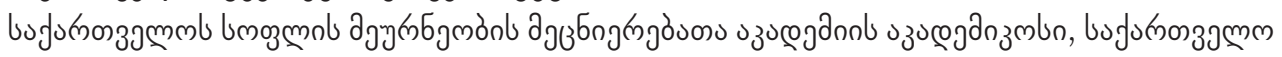

paata_koguashvili@hotmail.com

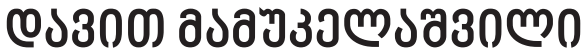

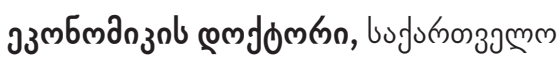

dmamukelashvili@gmail.com

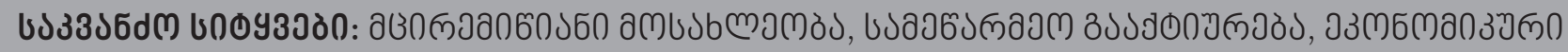

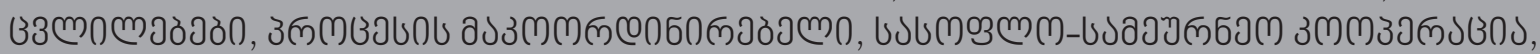

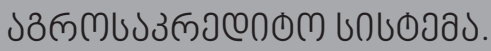

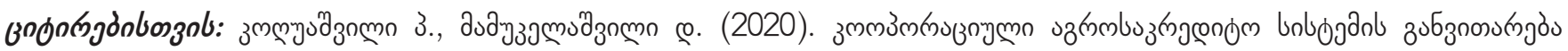

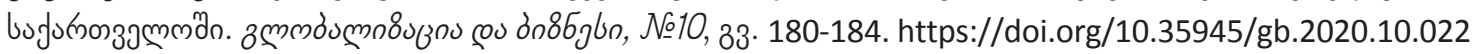

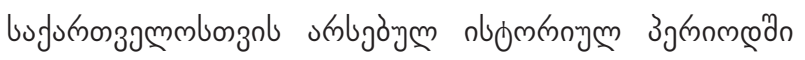

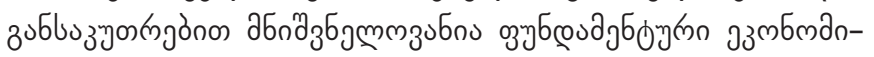

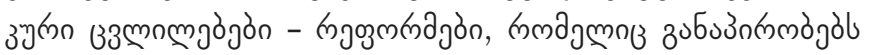

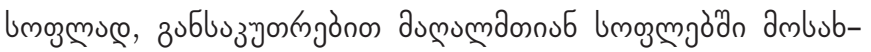

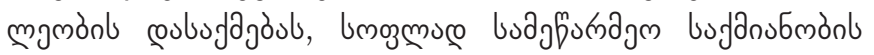

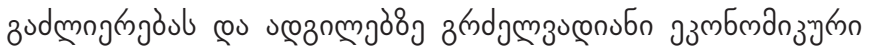

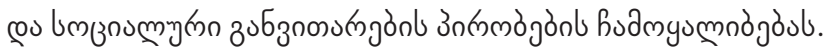

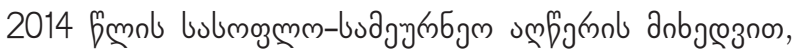

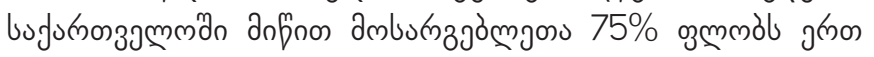

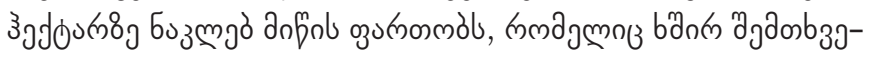

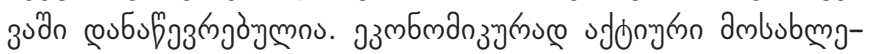

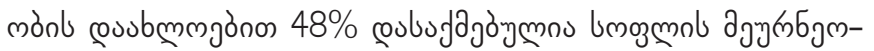
dudn, bucouz n

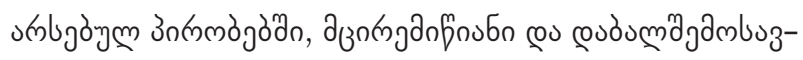

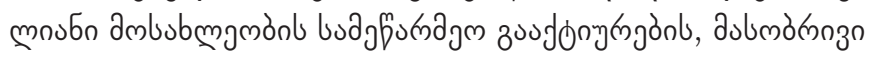

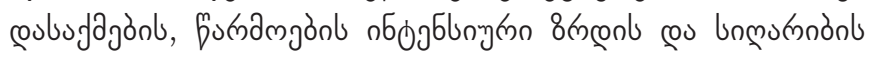

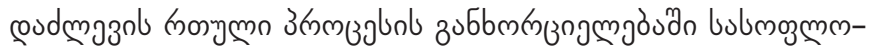
buajgn абпӘзб

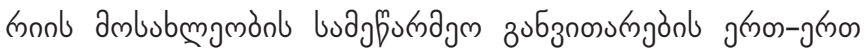

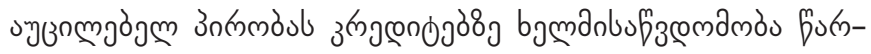

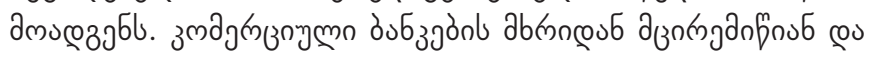

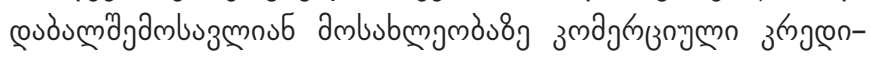

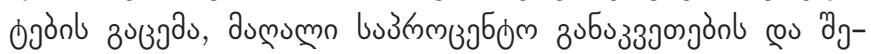

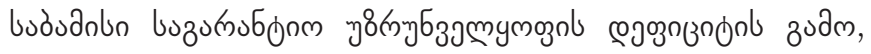

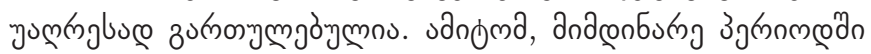




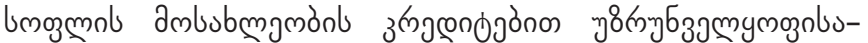

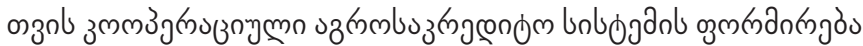

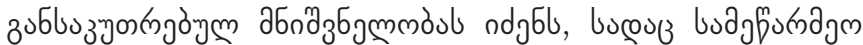

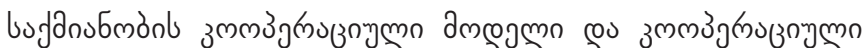

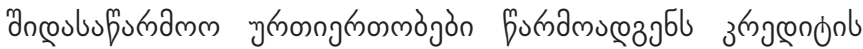

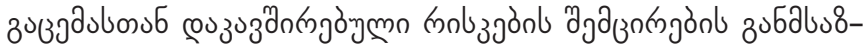

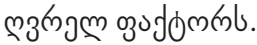

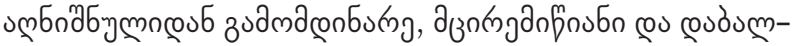

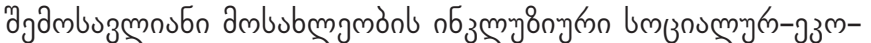

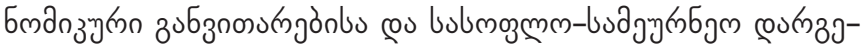

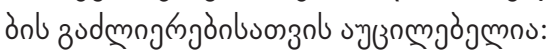

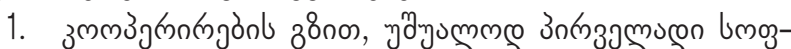

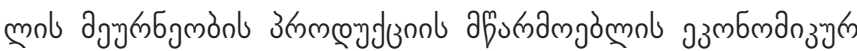
zudmngh

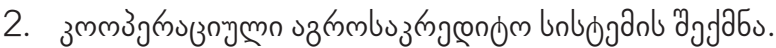

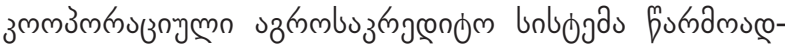

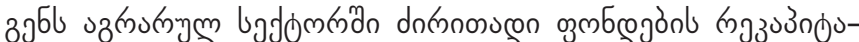

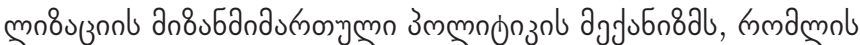

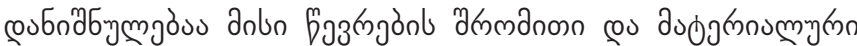

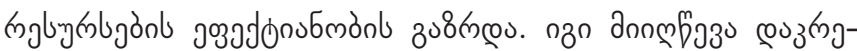

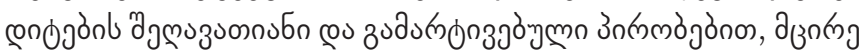

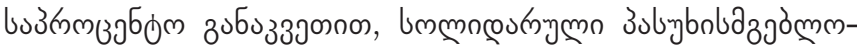

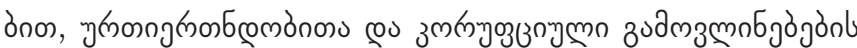

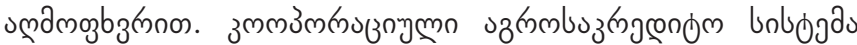

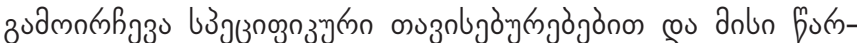

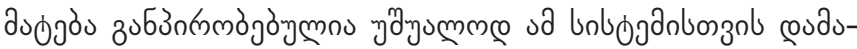

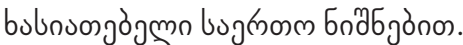

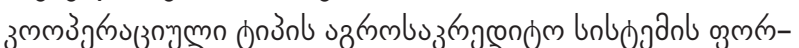
an

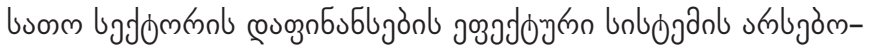

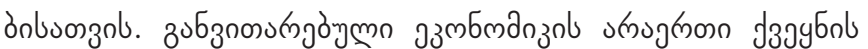

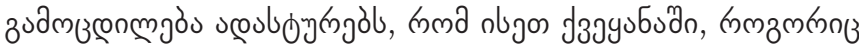

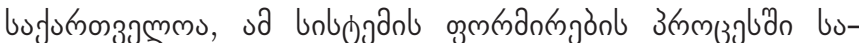

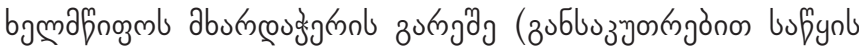

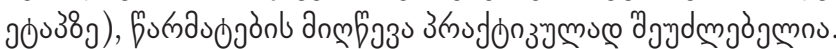

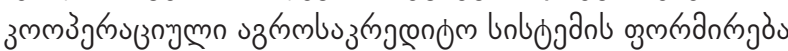

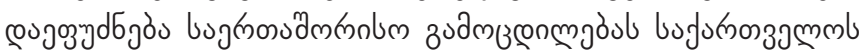

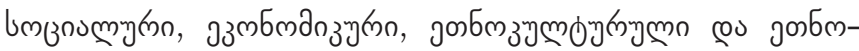

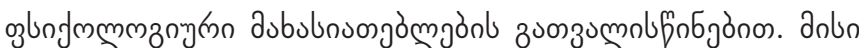

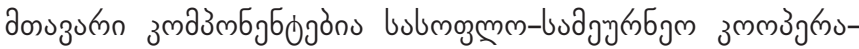

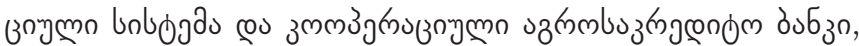

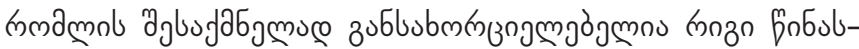

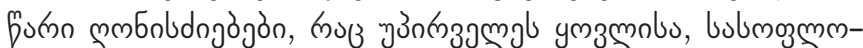
buagyn nб

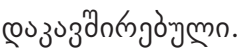

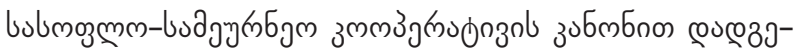

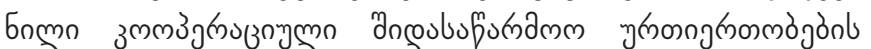

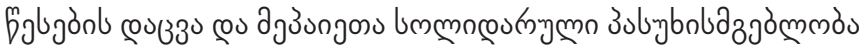

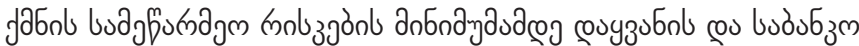

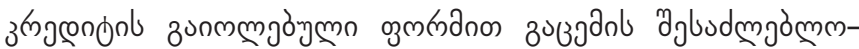

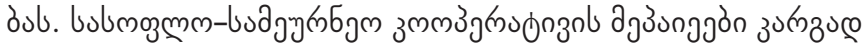

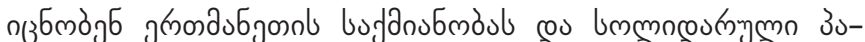

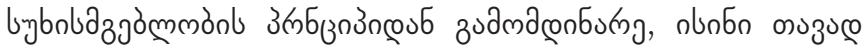

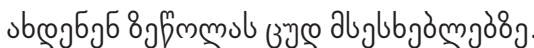

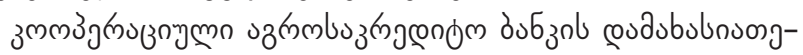

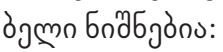

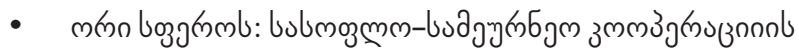

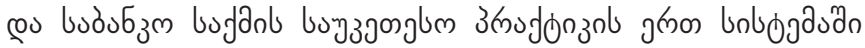

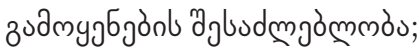

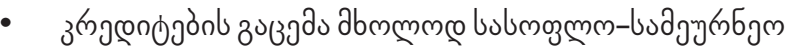

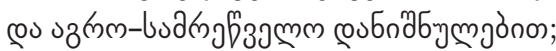

- z z з

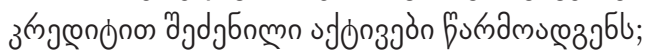

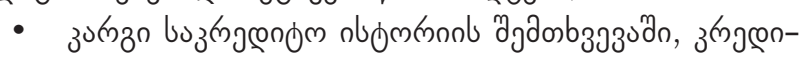

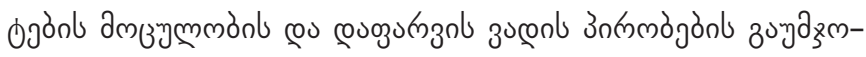

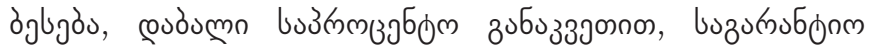

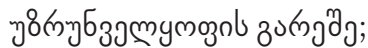

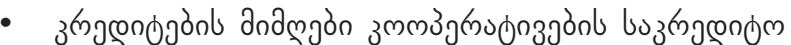
nbm

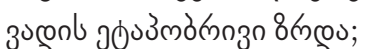

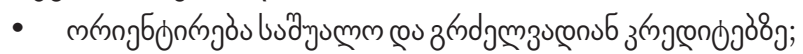

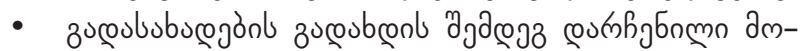

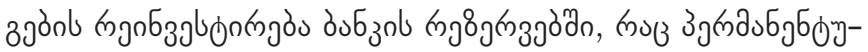

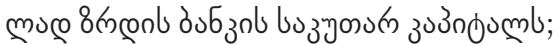

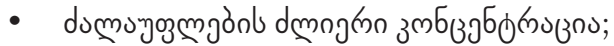

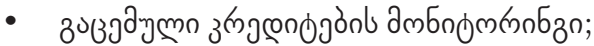

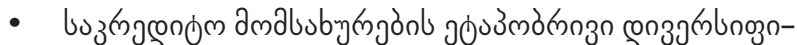
zongoso;

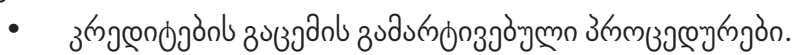

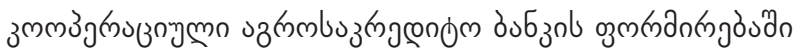

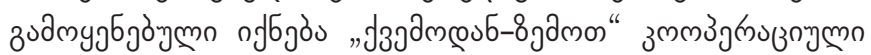

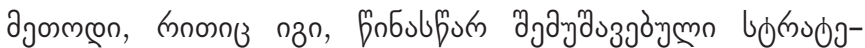
zחचm

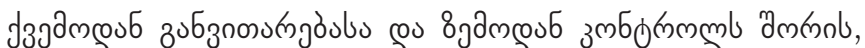

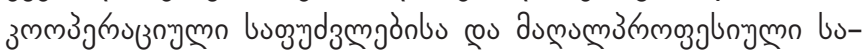

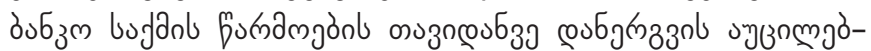
mmònl zuonzumolifn

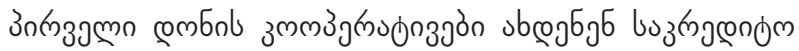

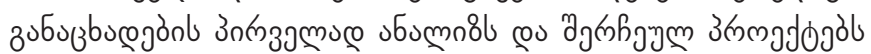

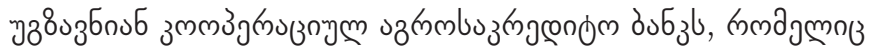

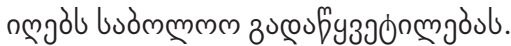

zubsozzumno

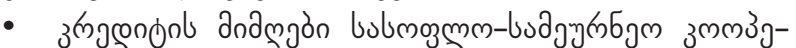

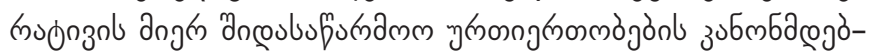

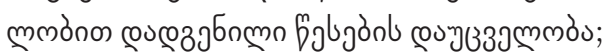

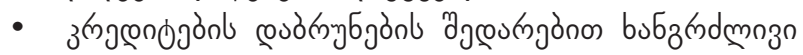
उुकू⿻m:

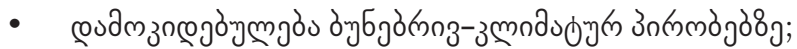

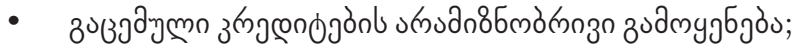

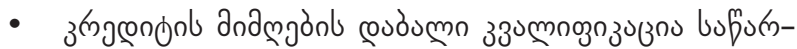




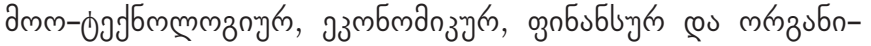

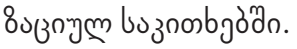

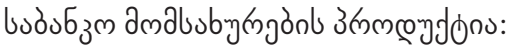

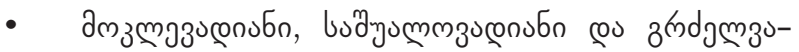

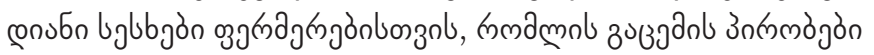

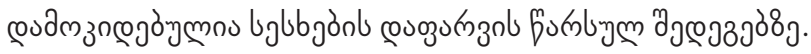

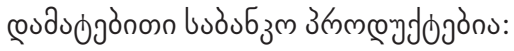

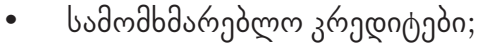

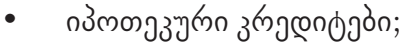

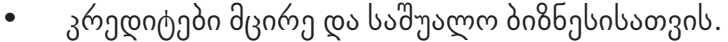

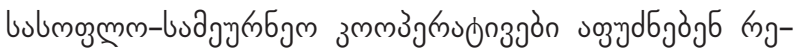

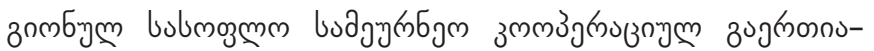

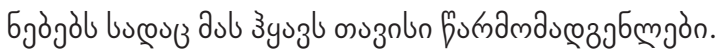

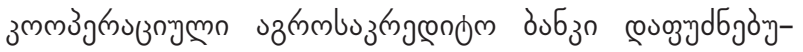

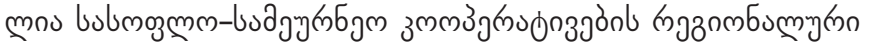

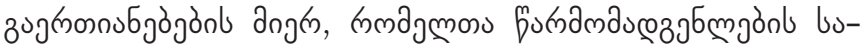

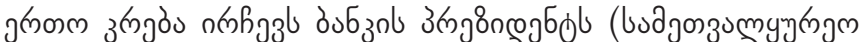

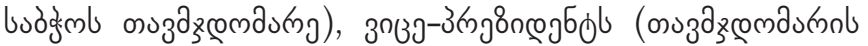

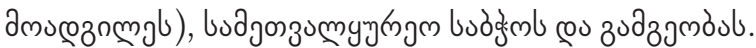

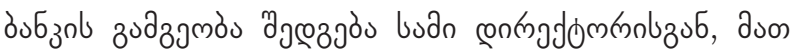

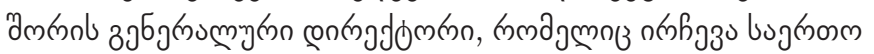

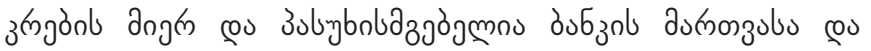

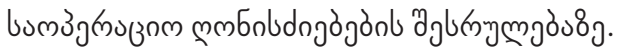

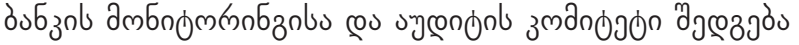

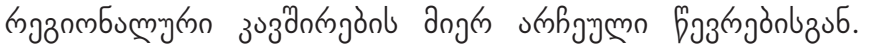
aub azumgdo du

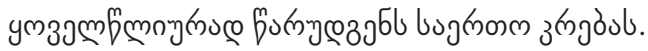

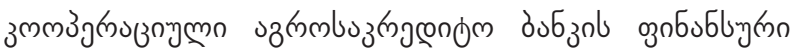

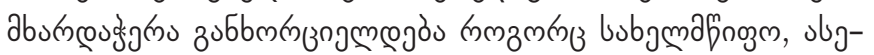

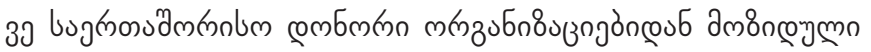
bublungònon.

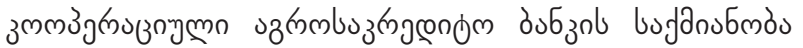
agndm

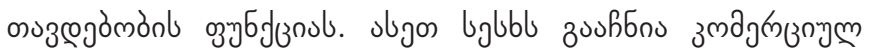

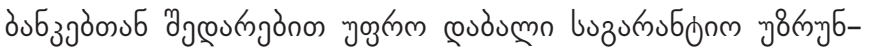

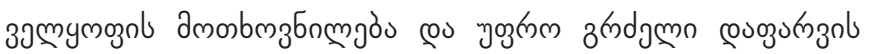

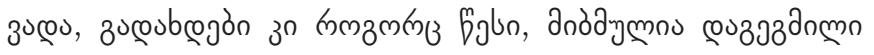

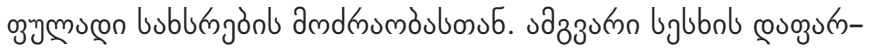

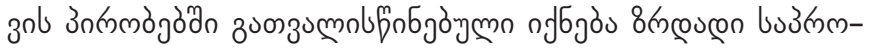

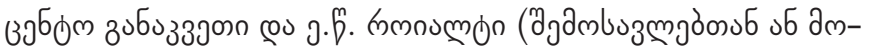

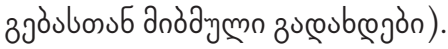

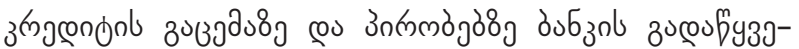

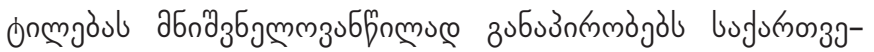

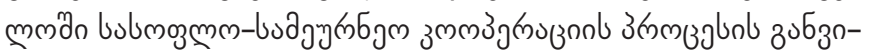

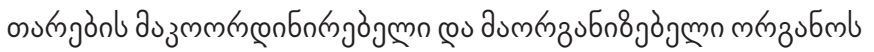

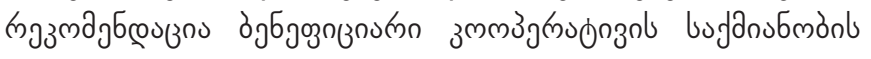

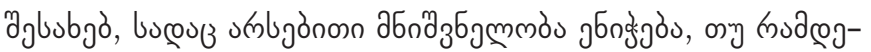

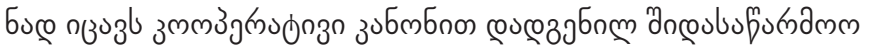

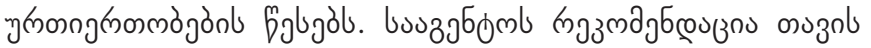

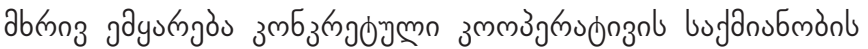

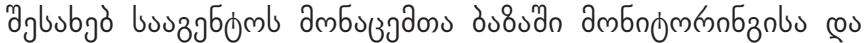

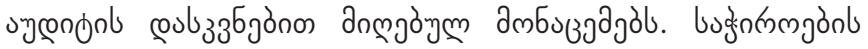

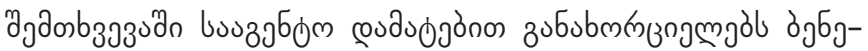

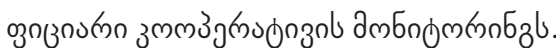

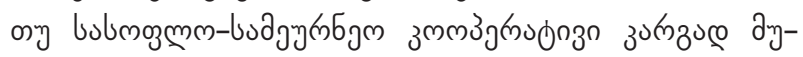
gumabl, उ उ

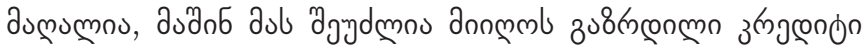

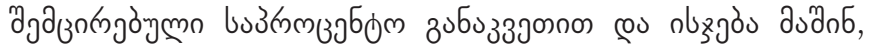

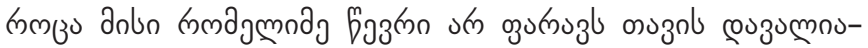

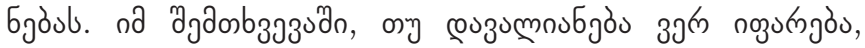

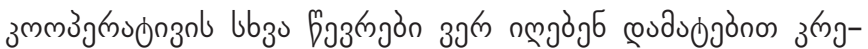

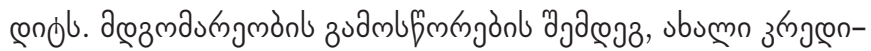

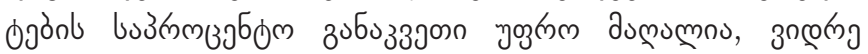

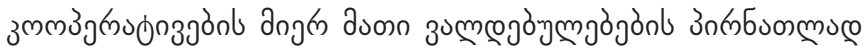

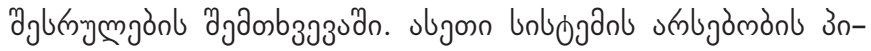

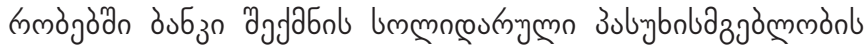

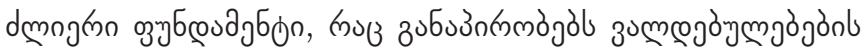

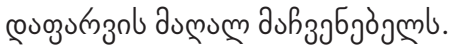

ügon oुm

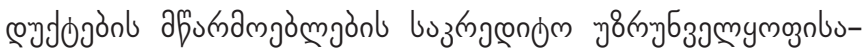

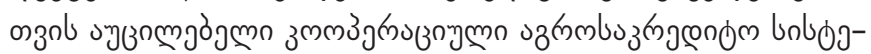
as, ذзау

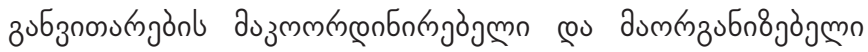

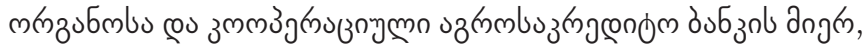

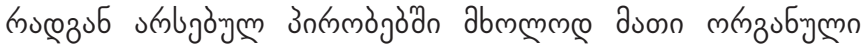

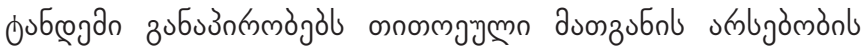

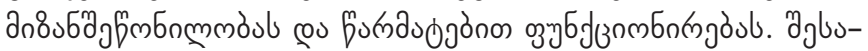

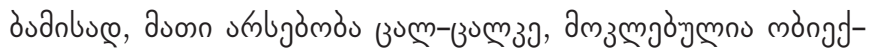
oyn bugydagmb.

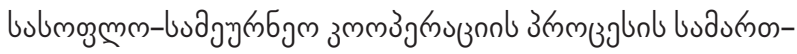

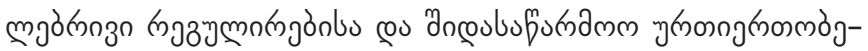

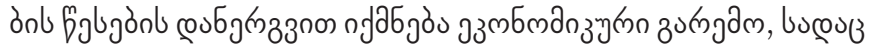

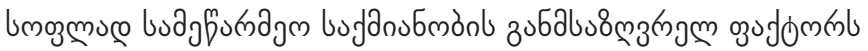
fumdmuœ

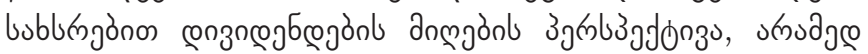

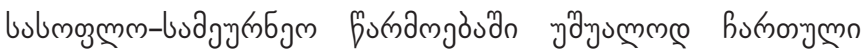

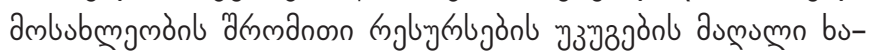

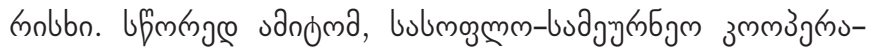

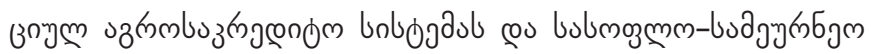

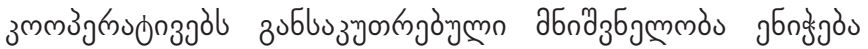

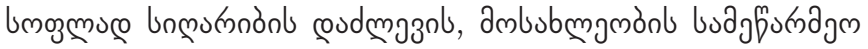

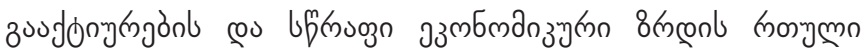

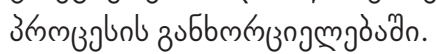

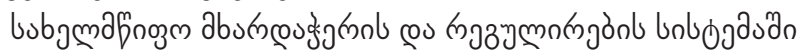

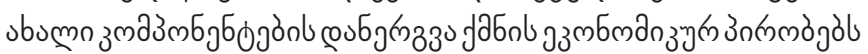

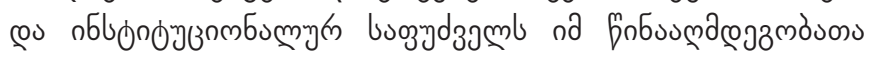

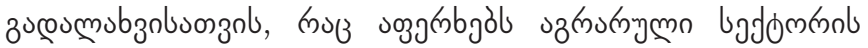

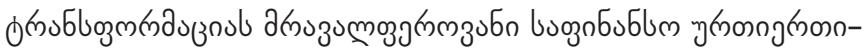

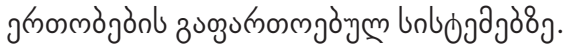




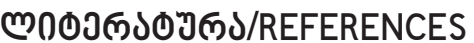

Cervera, E., Tudela, L., Cano, A., \& Guzman, S. (2017). Capacity Building to the Agriculture Cooperatives Development Agency (ACDA). 04/2017, Project Number: Europe Aid/136454/DH/SER/GE.

Koguashvili P. (2017). Investment system for financial support of cooperatives. Journal of New Agrarian Georgia. N4. (In Georgian) Mamukelashvili D. (2017). Legal regulation of agricultural cooperatives and rules of internal relations (training module). EU funded project. Europe Aid/136454/DH/SER/GE, 29/05/2017. (In Georgian)

Law of Georgia on Agricultural Cooperatives.12/06/2013. (In Georgian) 